\title{
Proteomic profiling of animal models mimicking skeletal muscle disorders
}

\author{
Philip Doran, Joan Gannon, Kathleen O'Connell and Kay Ohlendieck \\ Department of Biology, National University of Ireland, Maynooth Co. Kildare, Ireland
}

\begin{abstract}
Over the last few decades of biomedical research, animal models of neuromuscular diseases have been widely used for determining pathological mechanisms and for testing new therapeutic strategies. With the emergence of high-throughput proteomics technology, the identification of novel protein factors involved in disease processes has been decisively improved. This review outlines the usefulness of the proteomic profiling of animal disease models for the discovery of new reliable biomarkers, for the optimization of diagnostic procedures and the development of new treatment options for skeletal muscle disorders. Since inbred animal strains show genetically much less interindividual differences as compared to human patients, considerably lower experimental repeats are capable of producing meaningful proteomic data. Thus, animal model proteomics can be conveniently employed for both studying basic mechanisms of molecular pathogenesis and the effects of drugs, genetic modifications or cell-based therapies on disease progression. Based on the results from comparative animal proteomics, a more informed decision on the design of clinical proteomics studies could be reached. Since no one animal model represents a perfect pathobiochemical replica of all of the symptoms seen in complex human disorders, the proteomic screening of novel animal models can also be employed for swift and enhanced protein biochemical phenotyping.
\end{abstract}

Keywords:

Animal disease model / Animal proteomics / Biomarker / DIGE / Disease marker

\section{Introduction}

A major focus of modern biological research is to elucidate the molecular and cellular mechanisms that underlie human health and disease. Over the last few decades, both naturally occurring and genetically engineered animal models of human diseases have been employed for evaluating new therapeutic options and for elucidating pathological pathways [19]. In conjunction with findings from clinical investigations,

Correspondence: Professor Dr. Kay Ohlendieck, Department of Biology, National University of Ireland, Maynooth Co. Kildare, Ireland

E-mail: kay.ohlendieck@nuim.ie

Fax: +353-(1)-708-3845

Abbreviations: AK, adenylate kinase; DMD, Duchenne muscular dystrophy; EC, excitation-contraction; SR, sarcoplasmic reticulum
Received: January 15, 2007

Revised: March 1, 2007

Accepted: March 2, 2007 biological studies of animal models can be extremely helpful for the initial evaluation of alternative treatment strategies [10-12]. Genetic manipulations via transgenic or gene knockout technology can provide crucial information prior to the development of drug or gene therapy approaches to counter-act symptoms in human disorders [13, 14]. In addition, the potential outcome of cell-based therapies involving different kinds of stem cells can be mimicked through animal model research [15-17]. Currently, a variety of animal species are used for the detailed biochemical, cell biological and physiological analysis of almost all major human diseases [1-9], and are applied in essential toxicological investigations to determine the potential side effects of new pharmacological agents or treatment regimes $[18,19]$.

The traditional reductionist approach to investigating the pathobiochemical problems by focusing on single proteins, protein complexes or isolated pathways, has inevitably been criticized due to its limited scope in understanding biomedical phenomena at the systems level [20]. Although the 
last decades of biomedical research have produced a remarkable series of scientific breakthroughs on the elucidation of disease mechanisms, this vast biological knowledge had surprisingly little effect on the improvement of drug targeting. Although it is estimated that several hundred thousand different protein species or isoforms exist in the various human cell types, which represent a gigantic cohort of potential pharmacological targets, only a few hundred target-families are actually recognized by traditional drug discovery approaches [21]. Thus, detailed knowledge of a primary genetic abnormality or cellular mechanisms that underlie pathological processes does not necessarily translate into rationale drug design or improved cell-based therapies. Modern systems biology based on the graph and systems theory may succeed through mathematical modelling to deliver a better understanding of complex biological and pathological processes, but will heavily depend on reliable sets of data for producing relevant biomedical predictions [22]. In this respect, high-throughput genomic, proteomic and metabolomic technology will be an absolutely essential component in delivering these kinds of analytical results.

The tremendous technical advances in the highthroughput screening of peptides by MS have established a new analytical capability in the biological sciences [23]. It is now possible to identify large portions of the protein complement of a given cellular system in one swift analytical approach [24-26]. Naturally, the application of proteomics has also revolutionized the study of animal models mimicking human diseases. A better understanding of animal models of disease through genomics, proteomics and metabolomics is already clearly evident in the field of biomedicine [27-29]. Since the screening of patient samples usually requires very large experimental repeats due to considerable interindividual differences in humans, the usage of inbred and therefore genetically more homogeneous animal models is certainly advantageous in the initial search for new biomarkers of pathological processes. This allows animal model research in general and the proteomic profiling of species, that represent important aspects of human pathology, in particular a central place in modern biomedicine. Based on the findings from animal model proteomics, a more informed decision on the design of clinical proteomics studies could be achieved.

This review outlines the importance of studying animal disease models for enhancing our understanding of the fundamental pathological principles, critically examines the limitations of extrapolating animal data to the human subject and discusses how the identification of novel disease markers from animal studies can be used as a starting point for more in-depth medical investigations. It is clear from our current state of biomedical knowledge that it will be essential to develop better animal models that more closely resemble the various aspects of human pathology and to characterize already established animal strains in more detail. A more thorough biochemical analysis of animal models by comparative proteomics has an enormous potential to accelerate the process of improving diagnostic procedures and to decisively refine the development of new therapeutic strategies for the preventative and ameliorative treatment of all major classes of human disorders.

\section{Biomedical importance and pathobiochemical limitations of animal disease models}

Despite the ongoing ethical debate about whether it is justifiable to generate new, or manipulate established animal models, on purpose for biomedical research [30], it is clear that the biological analysis of animal models that mimic human diseases has led to a better understanding of numerous pathological mechanisms and was extremely helpful in the initial testing of new therapeutic strategies [1-9]. If the biological fact, that animal models usually do not represent a perfect replica of all pathobiochemical aspects seen in a highly complex human pathology, is properly taken into account, studying genetically engineered or naturally occurring species can yield important results of clinical relevance $[13,14]$. However, it is important to keep the limitations of animal experiments in mind and that the extrapolation of animal data to the human situation is sometimes very difficult due to species-specific differences. Small rodents commonly used in biological research may exhibit fundamental physiological differences as compared to humans. This includes differences in development, cellular differentiation, potential for tissue regeneration, mechanisms of cell regeneration, immune responses, susceptibility to infection, patterns of detoxification of xenobiotics, metabolism and bioenergetics. Variations in basic biological mechanisms such as the maintenance of ion homeostasis, cell signalling, hormonal regulation or metabolic integration may exist between cellular systems in animal models versus human patients. Furthermore, differences in compensatory mechanisms or redundant pathways between different species may complicate the interpretation of results from animal experiments. It is therefore essential to properly evaluate a new animal model prior to employing such a biological system in elaborate large-scale studies for testing new drugs or biomedical treatments. The biological integration of genomic, proteomic and physiological data sets from both human and animal model analyses will be an important aspect towards unravelling the fundamental differences between species [31, 32].

Taking into account the above outlined potential problems with biomedical data generated from animal experiments, a good animal model should exhibit the following characteristics. Ideally, it should (i) closely resemble the etiology of the human disease in onset, progression, complexity and severity, (ii) develop all or most of the multifactorial aspects usually observed in end-stage human pathology, (iii) mimic the basic mechanisms of human physiology and metabolism that are important for disease progression or drug treatment, (iv) exhibit similar primary 
genetic abnormalities in the case of inherited disorders, (v) show comparable susceptibility to infectious or pharmacological agents in the case of infectious or pharmacogenetic diseases, (vi) be suitable for genetic manipulations and cellbased treatment strategies and (vii) be large enough to yield sufficient amounts of biological samples for extended biochemical analyses or to facilitate physiological and surgical procedures $[1,2,10]$. The ease of breeding, housing and sampling are obviously other important criteria for choosing a suitable animal model. With respect to the humane and responsible use of animal models in biomedical research, an important bioethical objection is often associated with the usage of larger animals. This makes smaller rodents, especially genetic mouse models, the most frequently used species [3, 4, 14]. It is important to stress that different animal strains might be necessary for properly studying the various aspects of a multifactorial human disease.

\section{Animal model proteomics}

Although the usage of human cell cultures can give highly relevant data with respect to therapeutic dosage and toxic side effects [33], whole-animal studies will probably still form a central part of pharmacological and biomedical evaluations in the foreseeable future $[34,35]$. Cells in culture or co-culture often lack important aspects of differentiation and usually do not exhibit heterogeneous cell-cell interactions to the same degree as seen in tissues. In pharmacological studies, cell culture models cannot be employed to mimic the complexity of adsorption, distribution, first-pass hepatic elimination, modification and excretion of a drug. Besides the liver, biotransformation may involve many other tissues and can trigger severe organ-selective toxicity that can only be studied at the whole-body level [36, 37]. In the past, hypothesis-driven studies on animal models have focused on specific proteins, families of protein factors or pathways, greatly limiting the meaningfulness of experimental results with respect to understanding biological processes at the systems level. With the emergence of high-throughput proteomics technology, the biochemical characterization of animal models has been decisively improved. Technologydriven approaches in modern protein biochemistry has led to the development of analytical flow schemes for the fast and efficient identification of novel protein factors involved in disease processes.

Following the development of genetically engineered animal models or the discovery of naturally occurring disease models, the basic determination of genetic, physiological, histological and anatomical characteristics has to be carried out in order to judge the phenotype of a new experimental species. To properly mimic human symptoms, the onset, severity and progression of the disease should be similar in the animal model. In addition, basic physiological, metabolic and anatomical aspects also have to be taken into account when one judges the usefulness of an animal model.
Once a suitable biological system has been established, MSbased proteomic screening can be used to identify new candidate proteins with a changed expression level. While genomic data can only predict the effects of changes in gene expression on the actual concentration of proteins or the extent of PTMs, proteomics can accurately provide the status of protein abundance, isoform expression patterns and peptide modification. The large-scale proteomic profiling of proteins has enhanced the analytical capabilities to such an extent that it is now possible to study thousands of individual protein species in parallel [38-40]. As an illustrative example, see below the DIGE analysis of the dystrophic diaphragm from the mdx model of Duchenne muscular dystrophy (DMD) [41]. Soluble proteins can be conveniently studied by 2-DE [25, 42], while more insoluble membrane proteins are mostly analysed via LC [43]. MS-based proteomics is also highly suitable for determining important PTMs [44], which has established the high-throughput determination of disease-related changes in the phosphoproteome or the glycoproteome as a routine biochemical investigation.

Thus, in the modern biochemical laboratory, proteomic profiling represents the most powerful tool for the swift separation and identification of new biomarkers that exhibit altered expression levels in pathological tissues. Initial proteomics data should be confirmed by peptide sequencing, immunoblotting and/or immunofluorescence microscopy in order to establish a new cohort of potential disease marker proteins. For comparative searches, the existence of international data banks with standardized 2-D gel patterns of all major organ and tissue systems from established animal models can greatly simplify the identification of a new disease marker $[45,46]$. Once interesting candidate proteins have been found by animal model proteomics, an informed decision can be made on the optimum design of subsequent clinical studies. For example, instead of screening total extracts from human tissue specimens or separating the entire accessible protein complement, pathobiochemical studies can focus on particular subcellular fractions or only isolate subfractions with a specific biochemical property. If differential centrifugation steps, affinity isolation techniques or density gradient centrifugation methods do not lead to artefacts of enrichment or detachment of biomolecules, the analysis of low-abundance or relatively insoluble marker proteins should be best executed with subcellular fractions.

Groups of soluble human proteins that exhibit a restricted range of $\mathrm{p} I$-values and/or molecular masses, can be separated at a higher resolution by optimizing the IEF gradient and polyacrylamide concentration during 2-DE. If a new candidate protein has been shown to exist in large oligomeric clusters in healthy tissue, it might be worthwhile to evaluate its supramolecular status by intraproteomics. The fast isolation of large numbers of homogeneous protein species by preparative IEF or continuous gel electrophoresis, followed by conjugation to a signalling enzyme such as peroxidase, has the potential for being useful for high-throughput intraproteomics [47]. If a protein has been shown to be abnor- 
mally oligomerized or being of significant lower or higher concentration in an animal model, a simple comparative immunoblot analysis of age-and gender-matched normal versus pathological human biopsy material can quickly and efficiently give new insights into a disease process. Thus the initial discovery of new biomarkers by animal model proteomics, in conjunction with subsequent detailed clinical studies, has the potential for improving diagnostic procedures in the short-term and is helpful in the establishment of novel therapeutic targets in the long-term.

Since most animal models do not reflect all of the complex symptoms of a multifactorial human disease or do not properly mimic the severity or progression of the disorder [10], the usage of multiple strains of genetically engineered or natural species is often necessary [48]. In addition, in genetic diseases different tissues or organs may be affected to a varying degree in animal models versus patients. This makes the development of new disease models that exhibit specific aspects of a human disorder very important. An essential parameter during the production of new biomedical systems is the effective phenotyping of a new animal strain, so that a quick decision can be made on its pathological reliability. For example, the phenotyping of new genetic mouse models of diabetes has to take into account all aspects of diabetic complications, including the effects of obesity and related cardiovascular and kidney disorders, and be carried out by standardized high-quality metabolic and physiological tests [49]. In order to fully evaluate a metabolic syndrome in a genetically engineered mouse, testing would include measurements of general carbohydrate levels, whole-body glucose metabolism, lipids and cholesterol, as well as body composition, eating behaviour and activity. Important aspects are also food and fat absorption, and the response of the animal to interventions such as fasting, exercise or a high-fat diet. More specialized metabolic investigations would study the mechanisms of aberrant insulin signalling during hormonal resistance using isotopic measurements of intracellular metabolic pathway fluxes in peripheral organs, such as liver and muscle. In addition, metabolic changes on the level of the regulation of glycolysis versus gluconeogenesis, citric acid cycle fluxes, sources of plasma glucose, nutrient oxidation and futile metabolic cycle fluxes also have to be taken into account if one wants to fully understand the phenotype of a new animal model of diabetes [50-52].

\section{Proteomics of muscle degeneration}

Skeletal muscle represents the most abundant tissue in the human body and is involved in many essential physiological and biochemical processes, including movement, posture, heat homeostasis and metabolic integration [53]. Mechanical loading and neuromuscular activity play an important role in the maintenance of muscle fibre integrity. This is clearly illustrated by the effects of external electro-stimulation or fibre denervation on muscle mass and strength [54-56]. Termina- tion or changes at the essential nerve-muscle connection lead to drastic alterations in gene expression and consequently alterations in protein abundance and isoform patterns. Chronic low-frequency stimulation of a fast muscle induces a stepwise transition of the contractile apparatus, energy metabolism, ion homeostasis and regulation of the excitation-contraction-relaxation cycle [57]. Thus, the study of skeletal muscle pathology always has to take into account the important influence of the innervating motor neuron on the differentiation of the fibre population that constitutes a motor unit. The degeneration of skeletal muscles or abnormal muscle metabolism plays an important role in many human diseases, including both disorders with primary muscle pathology or those that indirectly affect the musculature via neuronal or metabolic abnormalities. Muscle diseases encompass inherited disorders such as X-linked muscular dystrophies of the type Duchenne or Becker, limbgirdle muscular dystrophies and congenital muscular dystrophy, acquired autoimmune disorders such as myasthenia gravis, and pharmacogenetic diseases such as malignant hyperthermia [53]. A typical neuromusclar disorder is motor neuron disease, which exerts a drastic secondary pathology on affected muscle fibres [53]. Extreme forms of the age-related decline in muscle function can also be considered a pathological process, whereby sarcopenia of old age is an excellent example of a highly complex multifactorial human disease. Recently, several studies on the proteomic profiling of animal models mimicking muscular dystrophy [41, 58-61] and sarcopenia [62-65] have been published. Since muscle fibres can be subdivided into different types of predominantly fast- or slow-twitching cells and because differentiated skeletal muscles remain highly plastic in their adaptive capabilities, the proteomics of fibre typing [66-69] and muscle transformation [70] has also been of considerable interest to cell biologists. Below we outline the results of these studies and describe the beginnings of skeletal muscle proteomics as a new biochemical field in basic and applied myology.

\subsection{Proteomic profiling of muscle fibre types}

To standardize the proteomic analysis of animal models with respect to a heterogeneous and highly plastic class of tissue such as skeletal muscles, it is important to establish the comprehensive 2-D reference maps of the proteome from the most frequently used experimental species. Reproducible 2-D spot patterns and the MS-based identification of the most abundant soluble muscle proteins has been carried out for two important biomedical species, the mouse [46] and the rat [71], whereby the overall distribution pattern of key muscle proteins is relatively comparable to the human fibre proteome [72, 73]. The proteomic profiling of fast versus slow muscles is crucial for the identification of reliable biomarkers that can differentiate between fibre type-specific disease processes. The transition of fibre types occurs during normal physiological adaptations to changed functional 
demands, such as extensive physical training or exposure to zero-gravity environments [74, 75], and in certain neuromuscular diseases or clinical treatments such as dynamic cardiomyoplasty [76]. The proteomic cataloguing of fibre type-specific muscle markers has been carried out for four animal species of importance for biomedical research, i.e. the mouse [67], the rat [69], the rabbit [70] and the pig [66]. These studies have confirmed the results from previous physiological and biochemical studies and have clearly established the various isoforms of the myosin heavy chain, myosin light chain, actin, troponins and tropomyosins as reliable indicators of fibre type distribution [66-70]. In addition, the proteomic analysis of animal muscles has lead to a better understanding of protein dynamics under conditions of rapid growth [77], neuromuscular junction formation [78] and exercise-induced changes in the muscle proteome [79] and mitochondrial differences between the heart and skeletal muscle [80].

Over the last few decades of skeletal muscle research, the view that muscles represent a homogeneous tissue with restrictive adaptable potential has dramatically changed [54]. It is now clear that skeletal muscles are highly versatile and can undergo a rapid transformation process involving transdifferentiation between different fibre types, the recruitment of the myo-selective stem cell pool of satellite cells and their subsequent innervation-dependent differentiation, and a certain degree of cellular destruction and regeneration cycles [56]. The chronic low-frequency stimulation of predominantly fast-twitching muscles, such as the rabbit tibialis anterior, can be used to investigate the stepwise responses of fast muscles to enhance neuromuscular activity under conditions of maximum activation [57]. Chronic conditioning triggers profound physiological, biochemical and histological changes including a slower twitch pattern of the contractile apparatus, an elevated aerobic-oxidative capacity, a drastically higher resistance to fatigue, smaller calibre cells, an increase in the half-relaxation time and the time-to-peak twitch tension, as well as the changes in the density and isoform expression pattern of proteins involved in the regulation of excitation-contraction (EC) coupling, ion homeostasis, the contractile machinery, metabolic integration and signal transduction [56, 57]. The biochemical alterations during the fast-to-slow transition process were confirmed by the MS-based proteomic analysis of chronic low-frequency stimulated rabbit fast muscles [70]. The observed changes in 16 identified biomarkers of muscle transformation have demonstrated that, besides myosin isoform switching, also an increased availability of oxygen for aerobic metabolism via myoglobin transport, the optimum utilization of free fatty acids via increased albumin transportation, a rearrangement of the creatine kinase isozyme pattern for enhanced mitochondrial activity, and the up-regulation of stress proteins for stabilising myofibrillar components with chaperones is crucial for muscle plasticity. Changes in glycolytic enzymes of the cytosol versus citric acid cycle components and other mitochondrial elements have been found to be suitable for determining fibre type shifting [70]. The transition of easily fatiguing muscle fibres into fatigue-resistant aerobic fibres appears to be an extremely complex biological process. Building on these encouraging findings, hopefully the application of the more discriminatory DIGE method will lead to the identification of a larger and better cohort of fibre type-specific marker proteins in the future.

\subsection{Animal models of muscle degeneration}

Although adult muscle wasting diseases are not as frequent as heart failure, liver disease or cancer, neuromuscular disorders play an important role in the field of inherited childhood diseases and in gerontology [53]. Since this review is concerned with animal model proteomics and because recent proteomic profiling studies have been carried out with dystrophic and aged muscles, we have focused our discussion on X-linked muscular dystrophy and sarcopenia of old age. Muscular dystrophies are a clinically well-characterized group of inherited muscle wasting disorders that can be subdivided based on their primary genetic abnormalities into X-linked Duchenne/Becker muscular dystrophy, EmeryDreifuss muscular dystrophy, limb-girdle muscular dystrophy, congenital muscular dystrophy, distal muscular dystrophy, Bethlem myopathy and myotonic dystrophy [81, 82]. Figure 1A shows the severe pathology of Duchenne muscular dystrophy. A transverse cryosection, stained with haematoxylin and eosin, exhibits fibres of abnormal rounded contour and with varying diameter. The histologically most striking feature is the infiltration of connective and fatty tissue, causing severe muscle weakness in Duchenne patients. A great variety of animal models exist for these muscular dystrophies including the X-linked dystrophic mdx mouse and its related mutants generated by genetic engineering or chemical mutagenesis, the Duchenne-like dystrophic dog model grmd, the dy mouse model of congenital muscular dystrophy, the various Sgc mice mimicking limb-girdle muscular dystrophies, and the Miyoshi myopathy-like SJL mouse with a deficiency in dysferlin. See the recent reviews for a comprehensive listing of animal models of muscular dystrophy [7, 16, 83-86].

The mdx mouse is a naturally occurring animal model of dystrophinopathy and exhibits many of the symptoms of muscle degeneration seen in human X-linked muscular dystrophy [87]. While $\mathrm{mdx}$ limb muscles exhibit relatively mild dystrophic changes [88], the diaphragm muscle is severely necrotic [89]. Skeletal muscles fibres from the mdx mouse have a point mutation in the dystrophin gene causing the near-to-total absence of the Dp427 isoform of this membrane cytoskeletal protein [90] and a concomitant reduction in dystrophin-associated glycoproteins such as dystroglycans (DGs), sarcoglycans (SG), syntrophins (SYN), sarcospan (SS) and dystrobrevins (DBs) [91]. In analogy to human dystrophic muscles, mdx fibres are characterized by varying degrees of necrosis, the appearance of regenerated myofibres with central nucleation, an increased susceptibility to os- 

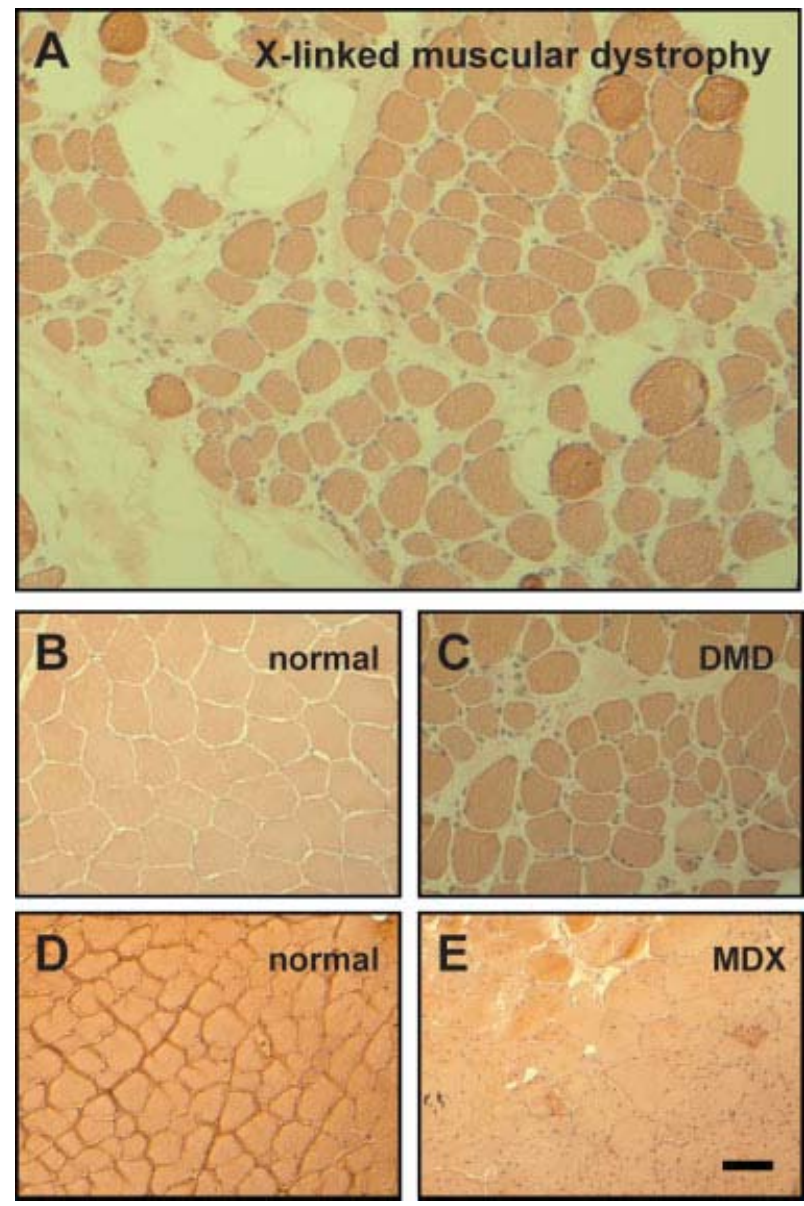

Figure 1. Histopathological comparison between DMD and the mdx mouse model of $X$-linked muscular dystrophy. Shown are $10 \mu \mathrm{m}$-thick transverse cryosections from human $(\mathrm{A}, \mathrm{C})$ and mouse (E) dystrophic skeletal muscle fibres and, for comparison, normal human (B) and mouse (D) muscles, respectively. Sections were stained with haematoxylin and eosin [91]. The specimens from the DMD patients exhibit signs of varying fibre size and infiltration of connective tissue. The $\mathrm{mdx}$ sample shows a high degree of central nucleation indicating repeated cycles of degeneration and regeneration. Bar $=100 \mu \mathrm{m}$.

motic shock, elevated levels of serum creatine kinase, stretch-induced injury, abnormal $\mathrm{Ca}^{2+}$-handling and impaired (EC) coupling [91-96]. Figure 1 shows the histopathological analysis of dystrophin-deficient fibres. The haematoxylin and eosin stained transverse sections show the dystrophic phenotype in a biopsy from a Duchenne patient versus the mdx mouse model of dystrophinopathy. In contrast to normal human and mouse skeletal muscle fibres (Figs. 1B and D), which show a regular distribution pattern and very little surrounding connective tissue, fibres from Duchenne muscle show severe dystrophic changes (Fig. 1C) and $\mathrm{mdx}$ fibres mildly dystrophic alterations with variations in cell diameter and extensive central nucleation (Fig. 1E). Interestingly, distal muscle groups in the eye and toe represent pro- tected phenotypes in the mdx mouse [97-99], which is possibly due to the up-regulation of the autosomal dystrophinhomologue named utrophin. The utrophin isoform Up395 is usually enriched at the neuromuscular junction [100], but found at high concentrations in extrajunctional sarcolemmal region in certain distal mdx muscles [97, 98]. Besides an utrophin-related rescue of the dystrophin-associated glycoprotein complex in extraocular muscles, possibly the small diameter and fast-twitch properties might result in a decreased loadbearing of these protected dystrophin-deficient fibres.

It is of considerable biomedical importance to elucidate the basic biochemical differences between severely necrotic diaphragm muscles, mildly dystrophic limb muscles and protected extraocular muscles from mdx mice. A differential effect of primary abnormalities on different subtypes of skeletal muscle is also present in human patients. It is not well understood why certain skeletal muscle groups are targeted or spared in a variety of neuromuscular diseases [81, 99]. Basic differences in molecular composition, physiological regulation, cellular structure and/or contractile properties may render fibres more or less susceptible to degenerative processes. In the case of the mdx mouse model of X-linked muscular dystrophy, the three main types of skeletal muscle are differentially affected [101]. Since the different fibre subtypes are present in the same animal model with the same primary genetic abnormality, compensatory mechanisms or physiological differences have to account for the variations in pathological features. Perhaps future comparative proteomics analyses of the three different mdx muscle types will shed light on these mechanisms and thereby identify new biomarkers involved in the natural rescue of the dystrophic phenotype [99]. The idea that full-length dystrophin and utrophin are functionally interchangeable isoforms and that increased utrophin levels are responsible for the milder $\mathrm{mdx}$ phenotype as compared to Duchenne patients, is supported by the analysis of the mdx/utrn ${ }^{-1-}$ mouse model [101-104]. This animal model of severe muscular dystrophy is deficient in both dystrophin and utrophin and exhibits weight loss, growth retardation, spinal curvature, joint contractures and premature death [101]. A comparative cataloguing of the muscle proteome of the $\mathrm{mdx}$ versus the $\mathrm{mdx} / \mathrm{utrn}^{-1-}$ mouse model of muscular dystrophy might be a useful starting point to explore the different patterns of fibre degeneration in the presence and absence of the Up395 isoform.

In addition to mammalian disease models, zebrafish and the nematode Caenorhabditis elegans have recently been used as nonmammalian models of muscle degeneration [86]. Both species express a dystrophin homologue and can be employed in large-scale genetic studies due to the ease of maintaining them in large numbers. Furthermore, their musculature and physiological control mechanisms are less complicated as compared to mammalian species, which makes them ideal high-throughput test systems for gene manipulation and pharmacological pilot studies [86]. The successful application of dystrophic models over the last few years has revealed the usefulness of animal models in 
applied biomedical research. New therapeutic strategies such as gene transfer methods (including viral infection, nonviral infection or antisense oligonucleotide approaches) or cellbased strategies (such as stem cell therapy or myoblast transfer) have all been tested in animal models of muscular dystrophy, mostly the mdx mouse [85]. Interestingly, the delivery of utrophin to neonatal $\mathrm{mdx} / \mathrm{utrn}^{-/-}$mice reduced the degree of necrosis in this animal model of severe muscular dystrophy [104], indicating that utrophin-based replacement therapies might be an option to treat progressive fibre degeneration in dystrophinopathies [105].

For the analysis of age-related muscle degeneration, various rat skeletal muscles are frequently used in biochemical studies. Muscle biopsy samples isolated from humans exhibit a high degree of interindividual differences, as recently shown in the analysis of vastus lateralis biopsies from male humans aged 18-82 years [106], making animal models of sarcopenia the preferred test system for initial proteomic studies. Established animal models of aging are represented by WI/HicksCar rats [107] and Fisher 344 rats [62]. The pathological profile of aged animals is often associated with other common aging-related diseases, such as diabetes, heart failure and cancer. It is, therefore, important to differentiate between unrelated aspects of pathophysiological mechanisms in the senescent organism. WI/HicksCar skeletal muscles exhibit age-related EC uncoupling and can be used as a suitable animal model of sarcopenia [107]. Depending on the particular rat strain, their average life span is between 24 and 34 months [62]. The majority of researchers in the field consider 4 months of age young, 12 months of age adult, 24 months of age old and 36 months of age senescent [64, $108,109]$. Below is a description of the proteomic profiling of skeletal muscles in animal models mimicking X-linked muscular dystrophy and sarcopenia of old age.

\subsection{Proteomic profiling of dystrophic $\mathrm{mdx}$ muscles}

Besides the proteomic analysis of immobilization-induced muscular atrophy in the rat $[110,111]$, fibre type changes in kyphoscoliotic mouse muscle [67], and age-dependent muscle wasting in rats [62-65], relatively little work has been done on the MS-based identification of pathological disease markers in animal models of neuromuscular diseases [112]. One exception is the X-linked muscular dystrophy [113], the most frequently inherited childhood disease of skeletal muscles [114], which has been chosen in this review as an illustrative example of the analytical power of modern proteomics [41, 58-61]. The reason for the extensive application of animal model proteomics in the case of DMD is probably the long-standing establishment of a variety of animal models mimicking this progressive disorder, as outlined above $[7,16$, 83-86]. The analysis of different groups of dystrophic muscles of varying age and pathological severity from the $\mathrm{mdx}$ mouse has identified several new disease markers, which have previously been missed by traditional biochemical approaches. It appears that the primary deficiency in the membrane cytoskeletal protein dystrophin causes a severe perturbation of the muscle proteome expression pattern including elements involved in nucleotide metabolism [58], cytosolic ion homeostasis [61], $\mathrm{Ca}^{2+}$-cycling through the sarcoplasmic reticulum (SR) [60], and the stress response via small heat shock proteins [41]. These proteomic findings can now be correlated to large data sets from various global gene expression studies with dystrophin-deficient fibres [115121]. Expression profiling suggests that cellular changes in dystrophin-deficient fibres are associated with altered developmental programming due to abnormal ion handling [115, $116]$, a chronic inflammatory response $[117,118]$, infiltration of connective tissue [118], an early delay in myofibre development [119] and a regeneration-inducing activation of signalling pathways involved in the proliferation and differentiation of satellite cells [120]. Interestingly, dystrophic muscle lacking both dystrophin and its autosomal homologue utrophin are characterized by the up-regulation of slow muscle genes [121], suggesting that these sets of genes may play a central role in the severely dystrophic phenotype. The results from both proteomic and transcriptomic profiling of dystrophin-deficient fibres agree that a large cohort of newly identified muscle components may represent potential targets for improved drug therapy. This includes elements of key metabolic pathways, ion homeostasis, stem cell activation, stress response and inflammation.

Figure 2 gives an overview of the findings from a recent DIGE analysis of the severely dystrophic diaphragm from mdx mice. Out of 2398 detectable 2-D protein spots, 35 proteins showed a drastic differential expression pattern, with 21 proteins being decreased, including Fbxo11-protein, adenylate kinase, haemoglobin and dihydrolipoamide dehydrogenase, and 14 proteins being increased, including aldehyde reductase, desmin, vimentin, stress proteins and myosin heavy chain isoforms [41]. This agrees with the idea that the lack of the dystrophin isoform Dp427 results in a destabilized membrane cytoskeleton and an increased frequency of microrupturing of the plasma membrane [122-124]. The expression pattern of key metabolic marker proteins involved in nucleotide metabolism, oxygen transportation, glycolysis, the citric acid cycle, fatty acid oxidation, aldehyde metabolism, the formation of acetyl-coenzyme, acid-base balance, ion homeostasis and the remethylation pathway are disturbed in the mdx animal model of Duchenne muscular dystrophy. The loss of metabolic regulators and compensatory up-regulation of key enzymes appears to be the pathobiochemical consequence of the interruption between the dystrophin-associated actin membrane cytoskeleton and the extracellular matrix (ECM) component laminin [122]. The up-regulation of the intermediate filament proteins vimentin and desmin in Dp427-deficient fibres is possibly a natural repair mechanism occurring in dystrophic fibres [41]. By substituting for the loss of dystrophin, the load-bearing function of the muscle cytoskeleton may be partially rescued by the increase in vimentin and desmin. The drastic reduction of the enzyme adenylate kinase (AK) in both the mildly 


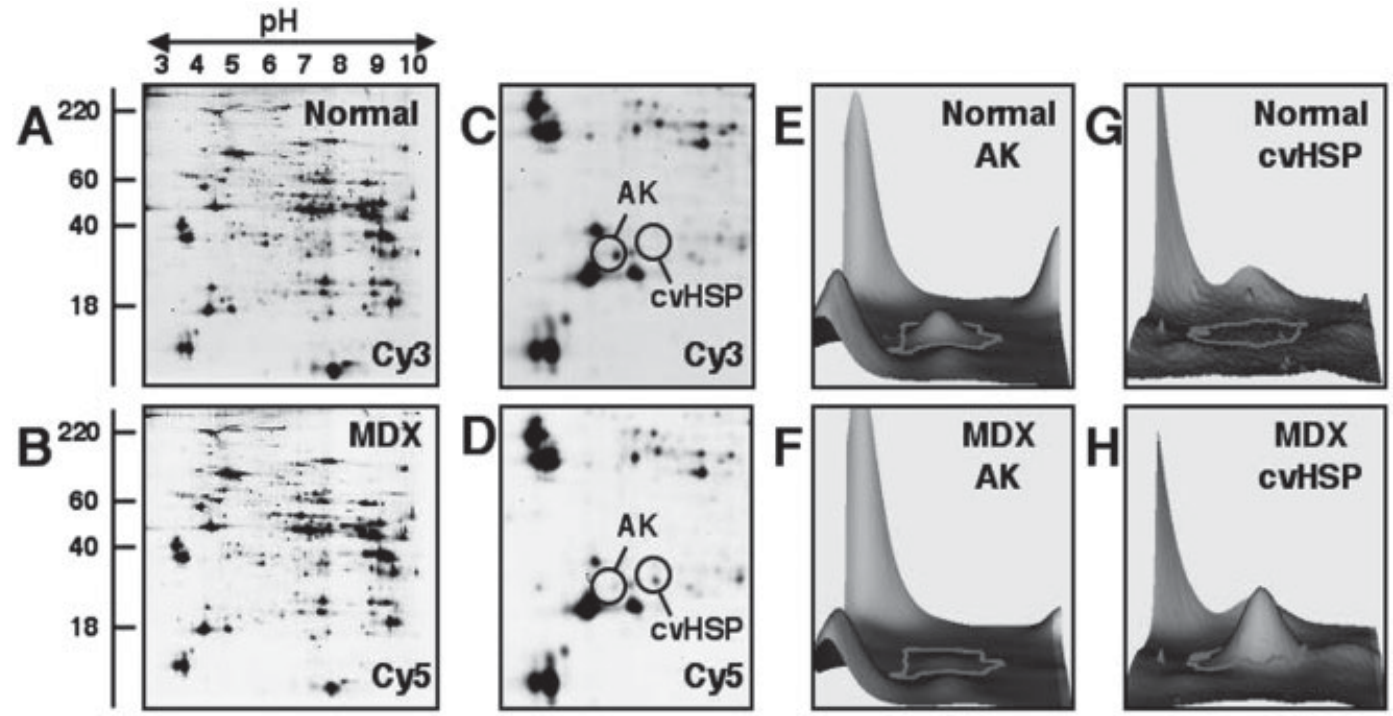

Figure 2. Proteomic profiling of the severely dystrophic mdx diaphragm using DIGE analysis. Shown is the 2-D gel electrophoretic separation of normal Cy3-labelled (A) and dystrophic Cy5-labelled (B) muscle preparations. DIGE analysis was carried out as previously described in detail [41]. The pH-values of the first dimension gel system and molecular mass standards (in $\mathrm{kDa}$ ) of the second dimension are indicated on the top and on the left of the panels, respectively. Panels (C) and (D) show an expanded view of 2-D gels of normal and $\mathrm{mdx}$ diaphragm, respectively. The position of the 2-D spots representing AK and the stress protein cvHSP are marked by circles. Changes in the abundance of these two protein in dystrophin-deficient fibres is illustrated in the diagrams of panels $(\mathrm{E})$ to $(\mathrm{H})$, demonstrating the drastic decrease of AK and concomitant increase of cvHSP in $\mathrm{mdx}$ fibres.

dystrophic mdx limb musculature [58, 59] and the severely dystrophic mdx diaphragm $[41,61]$, indicates a major metabolic defect in the nucleotide pathway.

The generally perturbed protein expression pattern in dystrophin-deficient fibres was found to be especially drastic with respect to a muscle-specific member of the small heat shock protein family [41], named cvHSP [125]. As can be seen in the enlargements of the Cy3- and Cy5-labelled 2-D gels of normal versus dystrophic preparations, the spots representing $\mathrm{AK}$ and cvHSP exhibit the opposite fate during muscle degeneration (Figs. 2A-D). The comparative graphic representation of the 2-D spots under investigation shows the drastic reduction in $\mathrm{AK}$ and concomitant increase in the expression of cvHSP in mdx diaphragm fibres (Figs. 2E-H). 2-D immunoblotting has confirmed these proteomic findings. Figure 3 shows the immunodetection of the stress protein cvHSP. In contrast to the unaltered abundance of the heat shock protein HSP60 and a decrease in the cystosolic $\mathrm{Ca}^{2+}$-binding protein regucalcin in $\mathrm{mdx}$ fibres, the expression of cvHSP is drastically increased in both young and old dystrophin-deficient fibres [41, 61]. The sub-family of small heat shock proteins is mostly involved in the stabilization of cytoskeletal protein folding and transportation which strongly indicates that the increase in cvHSP is an autoprotective mechanism of dystrophic fibres in an attempt to rescue their cellular integrity by modulating and repairing the cytoskeletal network [126, 127]. The above outlined findings from animal model proteomics suggest that modulating the stress response might be beneficial to damaged muscle

\section{Normal MDX}

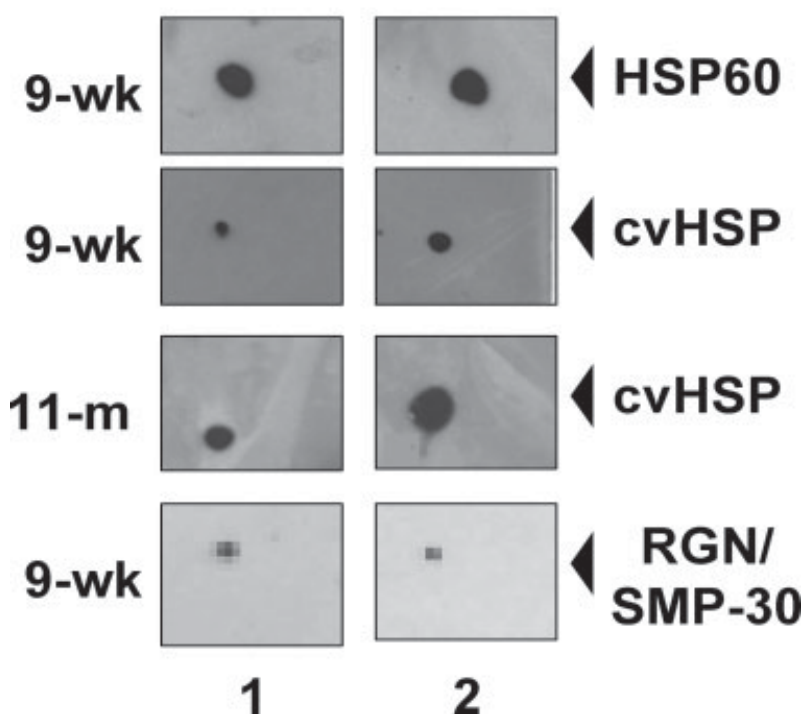

Figure 3. 2-D immunoblot analysis of the severely dystrophic $\mathrm{mdx}$ diaphragm. Shown are 2-D immunoblots of normal (lane 1) and dystrophic $\mathrm{mdx}$ (lane 2) preparations that have been labelled with antibodies to the heat shock protein HSP60, the small muscle-specific stress protein cvHSP and the cytosolic $\mathrm{Ca}^{2+}$-binding protein regucalcin ( $R G N$ ). Regucalcin is also named senescence marker protein SMP-30. The age of the muscles used in this analysis is marked at the left side of the blots (wk, weeks; $m$, months). 2-D immunoblotting was performed by standard methods as recently described by our laboratory [41]. 
fibres. Interestingly, heat shock treatment enhances the survival rate of transplanted myoblasts [128], demonstrating the important role of the stress response in muscle regeneration [129].

The calcium hypothesis of muscular dystrophy assumes that a combination of membrane rupturing, faulty repair mechanisms, abnormal ion fluxes and an impaired regulation of the excitation-contraction-relaxation cycle renders muscle fibres more susceptible to necrosis [130]. It is well established that $\mathrm{Ca}^{2+}$-cycling through the cytosol, sarcolemma, SR and mitochondria is disturbed in dystrophic fibres. The lack of dystrophin and resulting loss in the dystrophin-associated glycoprotein complex appears to damage the integrity of the skeletal muscle surface to such an extent that increased rupturing of the sarcolemma initiates a natu- ral membrane resealing process [131]. However, the introduction of sarcolemmal $\mathrm{Ca}^{2+}$-leak channels triggers a drastic elevation of cytosolic $\mathrm{Ca}^{2+}$-levels [132], which in turn causes proteolysis and thereby muscle degeneration [131]. A complicating factor is the decreased $\mathrm{Ca}^{2+}$-buffering capacity of the SR due to low levels of the $\mathrm{Ca}^{2+}$-binding proteins calsequestrin (CSQ) [60], CSQ-like proteins [95] and sarcalumenin (SAR) [96], and impaired cystosolic $\mathrm{Ca}^{2+}$-binding due to a reduction in regucalcin $[41,61]$. Since the cystosolic $\mathrm{Ca}^{2+}$. concentration is the most important second messenger system with respect to many muscle functions including EC coupling, fibre relaxation, metabolic integration and fibre differentiation, abnormal $\mathrm{Ca}^{2+}$-handling has severe pathological consequences [133]. The diagram in Fig. 4 outlines the current working hypothesis of the molecular pathogen-

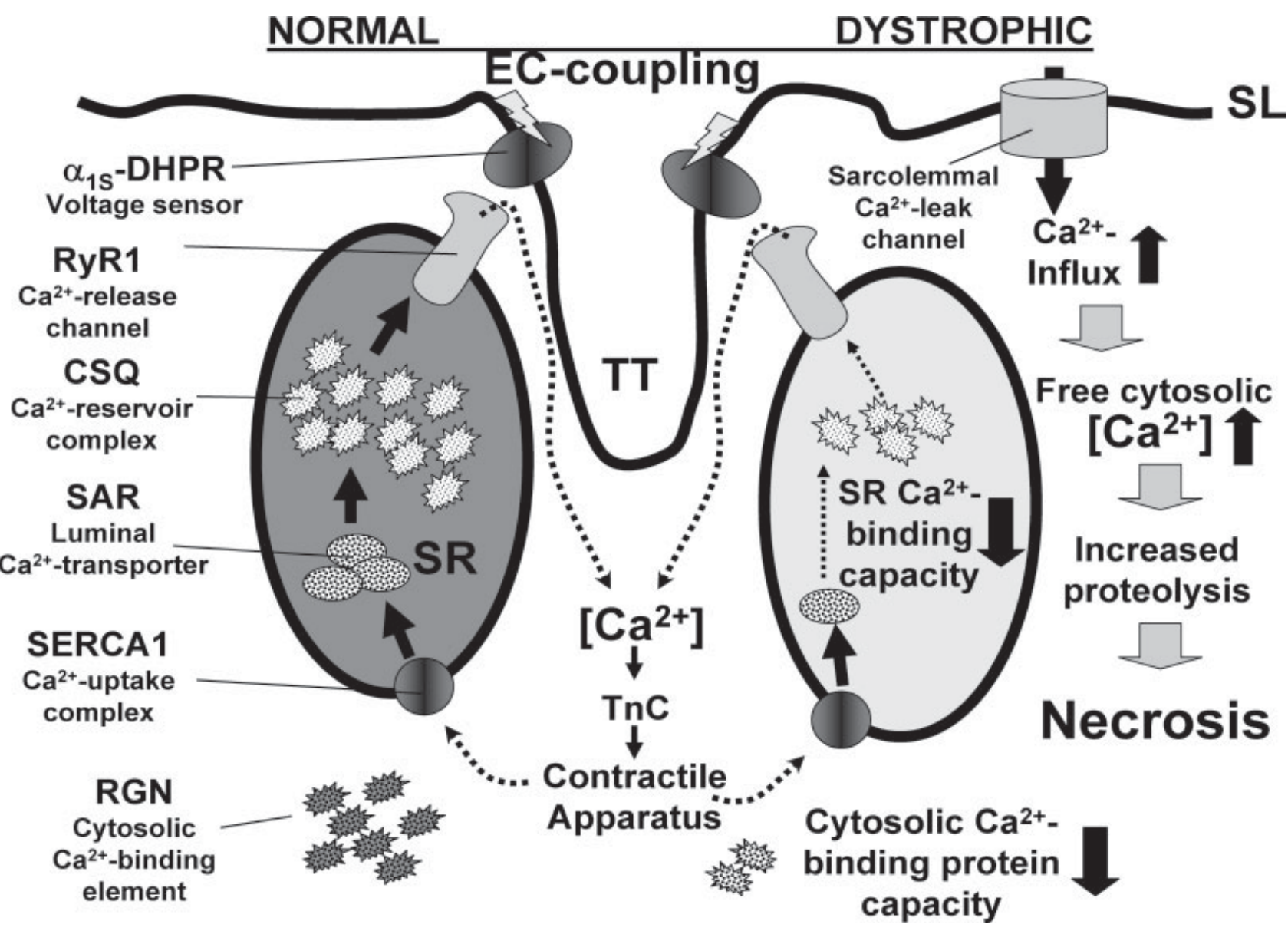

Figure 4. Overview of abnormal $\mathrm{Ca}^{2+}$-handling in dystrophin-deficient fibres as revealed by proteomic profiling of the mdx mouse model of DMD. Shown is a diagrammatic presentation of the main membrane systems involved in the regulation of the excitation-contractionrelaxation cycle. The contractile status of a skeletal muscle fibre is dictated by the cytosolic $\mathrm{Ca}^{2+}$-concentration, which regulates both contractile activity via the troponin $\mathrm{C}$ system $(\mathrm{TnC})$ and the relaxation-inducing pump activity of the sarcoplasmic reticulum $\mathrm{Ca}^{2+}$-ATPase (SERCA). Proteomic profiling of the $\mathrm{mdx}$ model has revealed that a reduced expression of the cytosolic $\mathrm{Ca}^{2+}{ }^{-b i n d i n g}$ protein regucalcin (RGN) and the luminal CSQ and SAR of the SR triggers abnormal ion homeostasis in dystrophic fibres. This results in a lower Ca ${ }^{2+}$-capacity of the luminal SR and the cytosolic ion-binding reservoir. In addition, abnormal EC coupling between the dihydropyridine receptor (DHPR) and the ryanodine receptor (RyR) $\mathrm{Ca}^{2+}$-release channel at the triad junction in the transverse tubules (TT) and the introduction of $\mathrm{Ca}^{2+}$-leak channels into the sarcolemma (SL) following membrane resealing exacerbates abnormal ion handling. Elevated cytosolic Ca ${ }^{2+}$-levels were shown to trigger an increased rate of proteolytic degradation of muscle proteins thereby rendering fibres more susceptible to necrosis. 
esis of muscular dystrophy based on the proteomic analysis of $\mathrm{Ca}^{2+}$-handling proteins. This included the subproteomic study of $\mathrm{Ca}^{2+}$-binding proteins by 'Stains All' labelling [60], the conventional proteomic profiling of soluble muscle proteins by Coomassie staining [61], and the comparative proteomic analysis of normal versus dystrophic fibres by DIGE screening [41].

Besides necrotic and regenerative muscle fibres, progressive muscle wasting in X-linked muscular dystrophy is also associated with an increase of endomysial fat and connective tissue, as well as infiltration by immune cells [ 81 , 114]. The analytical problem of dealing with nonmuscle cells can be addressed by careful prefractionation of tissue specimens or using the sensitive proteomic profiling technique itself for a differential analysis. High-resolution 2-DE in combination with MS identification tools is such a sensitive analytical approach that it can differentiate effects in heterogeneous cell mixtures. On the other hand, a prior manipulation of tissues, such as the extraction of different cell types from muscles by cell biological techniques, may introduce artefacts or more importantly decrease the amounts of available tissue for a proper proteomics analysis. Thus, despite the fact that tissue heterogeneity increases the complexity of a proteomic profiling exercise, a biochemical strategy using total muscle extracts should be successful for identifying new disease markers that play a central role in neuromuscular diseases. The screening of cell-specific biomarkers indicative of motor neurons, capillaries, connective tissue, fatty tissue and immune cells can be employed to judge drastic changes in these nonmuscle fibre cell types existing in muscles. In analogy to the identified changes in immune response and connective tissue composition as revealed by transcriptomic studies $[117,118]$, sensitive proteomic techniques such as DIGE analysis should be able to identify markers of fibrosis, inflammation and fatty tissue accumulation during muscle fibre degeneration.

\subsection{Proteomic profiling of muscle aging}

The comparative proteomic profiling of young versus old rat skeletal muscle by Piec et al. [65] revealed a differential expression pattern for various soluble proteins, such as elements of the contractile apparatus, the cytoskeleton, regulatory proteins, cytosolic and mitochondrial proteins involved in metabolism, and chaperones. The previous observation from biochemical studies of senescent muscles, that aging has a profound effect on many essential cellular processes [134], was confirmed by animal model proteomics. Sarcopenia appears to be associated with changes in the expression levels of key muscle proteins including actin, myosin light chains, troponin, desmin, tubulin, gelsolin, triose-phosphate isomerase, enolase, glycerol-phosphate dehydrogenase creatine kinase, isocitrate dehydrogenase, cytochrome-c oxidase, aldehyde dehydrogenase, glutathione transferase, glyoxalase, RNA-editing proteins and various chaperones [65]. These severe alterations may play a central role in the dysfunctions in aged muscle force generation and speed of contraction. It is believed that a variety of biochemical and cell biological factors play a role in the functional decline of aging muscles and it is difficult to determine whether a molecular pathological hierarchy and/or interdependence exists between the various metabolic insults or if one overriding pathological change triggers a cascade of pathophysiological down-stream responses [135]. Since the biological changes observed following denervation of a motor unit are similar to the natural muscle aging process, sarcopenia might be at least partially due to abnormal innervation. This would be in agreement with the generally observed loss of active motor units and a shift to a slower twitching phenotype in aged muscles [136, 137]. Besides dennervation, other factors involved in the loss of muscle mass and strength is the decreased protein synthesis of contractile muscle proteins, a progressive deterioration in energy intake, mitochondrial decline leading to an impaired energy supply to muscle cells, a disturbed equilibrium of growth factors and hormones essential for the maintenance of basic muscle function, modified gas and metabolite exchange rates due to decreased capillarization, extensive oxidative stress, increased triggering of apoptotic pathways, impaired ion handling leading to disturbed ion fluxes, abnormal metabolic regulation and a decreased availability of satellite cells [138-145]. Future DIGE-based analyses of aging muscle will hopefully identify a new range of biomarkers associated with sarcopenia. Once new aging-related protein factors have been shown to play a critical role in senescent muscle degeneration, novel therapeutic routes can be designed to counter-act one of the most devastating pathologies of old age.

\section{Impact of animal studies on clinical proteomics}

Although the identification of the primary genetic abnormality in an inherited disease or the determination of genetic factors that may predispose an organism to a certain pathology are in themselves remarkable scientific achievements, this biomedical knowledge does not necessarily translate into the quick development of a cure for these disorders. The human body consists of billions of cells forming a highly complex and metabolically integrated system that reacts to a genetic or environmental insult through the utilization of compensatory mechanisms and activation of alternative pathways. It is very difficult to predict at what point in time during a pathophysiological process the natural defence and repair mechanisms are exhausted and severe cellular damage starts to occur. Metabolic changes are often complex and a hierarchy may exist in degenerative pathways. These biological alterations are reflected in the constant change of the proteome during a patient's lifetime. Thus, in contrast to the limited information one can deduce from genomic data with respect to disease progression, the analysis of changes in the 
protein complement of an affected tissue or organ over time may give important clues on secondary and tertiary alterations. However, most human diseases show a whole array of symptoms with interindividual differences in severity and progression, making it often difficult to identify disorderspecific biomarkers. This gives animal model research an important place in biomedical studies. To outline the potential impact of animal proteomics studies on subsequent clinical proteomic profiling exercises, the findings from the DIGE screening of the mdx mouse model of DMD are taken as an example [41] and discussed with respect to their biomedical importance.

In the case of Duchenne muscular dystrophy, it has been known for almost two decades that point mutations or genetic rearrangements in the dystrophin gene, located in the chromosomal Xp21 region, are the underlying reason for progressive muscle wasting [146]. The enormous size and complexity of the dystrophin gene is possibly a major factor for the high frequency of this neuromuscular disease in young boys. Extensive biochemical studies on the function of dystrophin and its associated surface glycoproteins indicate that the loss of the linkage between the actin membrane cytoskeleton and the ECM is the initial cellular damage in dystrophin-deficient fibres [122]. A drastic reduction in the sarcolemma-spanning dystrophin-DG complex severely impairs the integrity of the muscle periphery [123]. It is not known how many secondary steps lead from this weakening of the plasma membrane to triggering the end-stage dystrophic phenotype with necrosis, fibrosis and fatty tissue infiltration [147]. Although abnormal $\mathrm{Ca}^{2+}$-handling has clearly been identified as a major factor in rendering muscle fibres more susceptible to proteolytic degradation, it is not well understood how altered ion fluxes trigger complex changes in metabolic regulation and signal transduction processes. On the level of the muscle fibre, necrosis is clearly the key mechanism which causes a decline in muscle mass and strength in patients suffering from X-linked muscular dystrophy. However, several years of a symptom-free stage is present in very young patients, suggesting that compensatory mechanisms based on the up-regulation of dystrophin homologues and/or the rescue by satellite cell proliferation exists. Once the body starts to loose sufficient numbers of muscle precursor cells, dystrophic changes will inevitably lead to muscle wasting. The missing link between the initial cellular defect via the loss of the dystrophin-glycoprotein complex and the final disintegration of muscle cells is the molecular pathogenesis bridging these two events [123].

As outlined in the flow chart of Fig. 5, the recent DIGE analysis of the severely dystrophic diaphragm from the $\mathrm{mdx}$ mouse model of DMD has revealed a large number of biological processes being affected in dystrophin-deficient fibres. Muscle proteins of increased abundance can be grouped as components involved in chaperone function (cardiovascular heat shock protein; chaperonin), aldehyde metabolism (aldehyde reductase), intermediate filament formation (desmin; vimentin), muscle contraction (myosin
X-linked muscular dystrophy

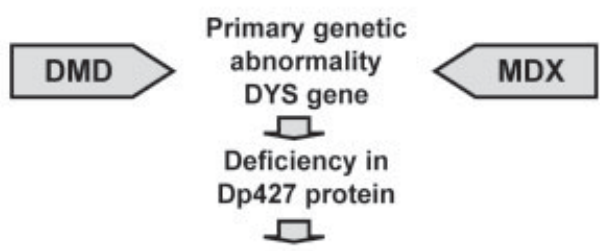

Loss of dystrophin-associated glycoprotein complex
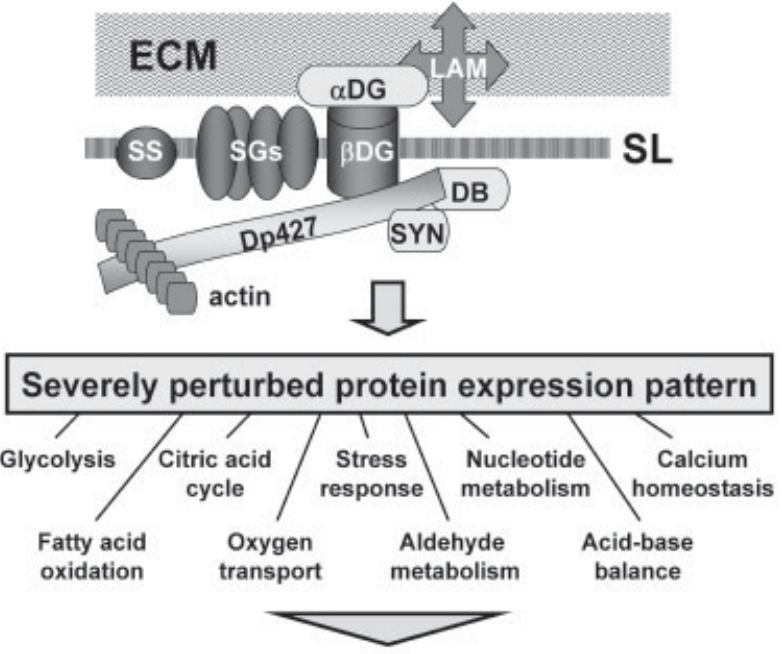

\section{New cohort of biomarkers of dystrophinopathy}

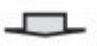

\section{Clinical evaluation in DMD muscles}

Figure 5. Flow chart of biomarker identification for muscular dystrophy. Shown is a diagram of the dystrophin-glycoprotein complex and its role in dystrophinopathies. Both, in DMD and the dystrophic mdx mouse model, the Dp427 isoform of the membrane cytoskeletal protein dystrophin is absent, triggering a reduction in the dystrophin-associated glycoprotein complex. In normal muscle, the $\alpha / \beta-D G$ subcomplex provides a trans-sarcolemmal linkage between the ECM element laminin (LAM) and the actin membrane cytoskeleton. Other proteins that exist in close proximity to dystrophin are DB, SYN, SS and the SG. The proteomic profiling of the $\mathrm{mdx}$ mouse model of $\mathrm{X}$-linked muscular dystrophy has resulted in the establishment of a new cohort of potential biomarkers of DMD. The biological processes, in which proteins with a disturbed expression pattern are involved in, are listed in the diagram.

heavy chains), glycolysis (glyceraldehyde-3-phosphate dehydrogenase), fatty acid oxidation (electron transferring flavoprotein) and nucleotide metabolism (Atp5b protein). In contrast, diaphragm proteins of decreased abundance are involved in protein ubiquination (Fbxo11 protein), nucleotide metabolism (adenylate kinase; creatine kinase), oxygen transport ( $\beta$-haemoglobin; $\alpha$-globin), mitochondrial enzymes associated with the formation of acetyl-coenzyme A (dihydrolipoamide dehydrogenase), muscle contraction (myosin light chain; troponin; actin), glycolysis (aldolase), 
aldehyde metabolism (aldehyde dehydrogenase), the remethylation pathway of homocysteine homeostasis (betaine-homocysteine methyltransferase), cytosolic calcium homeostasis (regucalcin), the polyol pathway of glucose metabolism (sorbitol dehydrogenase), the citric acid cycle (isocitrate dehydrogenase), the regulation of acid-base balance (carbonic anhydrase), and transcriptional control (jumonjidomain containing Jmjd1a protein) [41]. The disease-specific character of this new cohort of potential biomarkers of dystrophinopathy can now be tested by immunoblotting, immunofluorescence microscopy and comparative proteomics of human biopsy specimens, showing the usefulness of animal model proteomics as an initial enabling tool for clinical proteomics.

\section{Conclusions}

Based on the enormous impact proteomics had on biomedical studies over the last few years [148-150], it can be expected that the proteomic profiling of animal disease models will have a considerable influence on the improved design of diagnostic procedures, the evaluation of experimental treatment strategies such as stem cell and gene therapy, and the detailed biochemical phenotyping of new disease model systems. Since end-stage pathologies may involve similar degenerative pathways, it is important to distinguish between common indicators of general cellular destruction and subsets of more precise markers of a particular disease. Such knowledge can then be used to decisively improve the identification of new therapeutic targets. Since most complex human diseases are multifactorial and affect more than one tissue or organ, in the future multiple animal models mimicking particular aspects of a human pathology will be employed in order to properly study all aspects of a particular etiology. Thus, the genetic engineering of animal models will play an essential role in the study of the molecular and cellular changes caused by a genetic or environmental insult. Besides genetic rodent models, also nonmammalian systems such as zebrafish and nematodes will probably become more important due to the ease of genetic manipulation and analysis of physiological changes. In this respect, the ultimate goal of animal model proteomics is to facilitate the establishment of improved disease models and the identification of novel highly disease-specific biomarkers which can be employed for the predictive evaluation of new treatment strategies for human diseases.

The laboratory of the authors is supported by a principal investigator grant from Science Foundation Ireland (SFI-04/ IN3/B614) and project and equipment grants from the Irish Health Research Board (HRB-RP/2005/3; HRB-EQ/2003/3; HRB-EQ/2004/2). We thank Muscular Dystrophy Ireland for their continuous support of our research into neuromuscular diseases.

\section{References}

[1] Monnet, E., Chachques, J. C., Animal models of heart failure: What is new? Ann. Thorac. Surg. 2005, 79, 1445-1453.

[2] Hansen, K., Khanna, C., Spontaneous and genetically engineered animal models; use in preclinical cancer drug development. Eur. J. Cancer 2004, 40, 858-880.

[3] Glasser, S. W., Nogee, L. M., Genetically engineered mice in understanding the basis of neonatal lung disease. Semin. Perinatol. 2006, 30, 341-349.

[4] Joshi, R. L., Lamothe, B., Bucchini, D., Jami, J., Genetically engineered mice as animal models for NIDDM. FEBS Lett. 1997, 401, 99-103.

[5] Belanger, M., Butterworth, R. F., Acute liver failure: A critical appraisal of available animal models. Metab. Brain Dis. 2005, 20, 409-423.

[6] Uversky, V. N., Neurotoxicant-induced animal models of Parkinson's disease: Understanding the role of rotenone, maneb and paraquat in neurodegeneration. Cell Tissue Res. 2004, 318, 225-241.

[7] Allamand, V., Campbell, K. P., Animal models for muscular dystrophy: Valuable tools for the development of therapies. Hum. Mol. Genet. 2000, 9, 2459-2467.

[8] Patel, M., Goldstein, H., Animal models of cancer and HIV. Curr. Opin. Oncol. 2004, 16, 463-467.

[9] Phillips, A., Janssen, U., Floege, J., Progression of diabetic nephropathy. Insights from cell culture studies and animal models. Kidney Blood Press. Res. 1999, 22, 81-97.

[10] Russell, J. C., Proctor, S. D., Small animal models of cardiovascular disease: Tools for the study of the roles of metabolic syndrome, dyslipidemia, and atherosclerosis. Cardiovasc. Pathol. 2006, 15, 318-330.

[11] Hawk, E. T., Umar, A., Lubet, R. A., Kopelovich, L., Viner, J. L., Can animal models help us select specific compounds for cancer prevention trials? Recent Results Cancer Res. 2005, 166, 71-87.

[12] Maronpot, R. R., Flake, G., Huff, J., Relevance of animal carcinogenesis findings to human cancer predictions and prevention. Toxicol. Pathol. 2004, 32, 40-48.

[13] Nomura, T., Practical development of genetically engineered animals as human disease models. Lab. Anim. Sci. 1997, 47, 113-117.

[14] Sharpless, N. E., Depinho, R. A., The mighty mouse: Genetically engineered mouse models in cancer drug development. Nat. Rev. Drug Discov. 2006, 5, 741-754.

[15] Almeida-Porada, G., Zanjani, E. D., A large animal noninjury model for study of human stem cell plasticity. Blood Cells Mol. Dis. 2004, 32, 77-81.

[16] Wells, D. J., Wells, K. E., What do animal models have to tel us regarding Duchenne muscular dystrophy? Acta Myol. 2005, 24, 172-180.

[17] Carver, B. S., Pandolfi, P. P., Mouse modeling in oncologic preclinical and translational research. Clin. Cancer Res. 2006, 12, 5305-5311.

[18] Meneses-Lorente, G., Guest, P. C., Lawrence, J., Muniappa, N. et al., A proteomic investigation of drug-induced steatosis in rat liver. Chem. Res. Toxicol. 2004, 17, 605-612.

[19] Meneses-Lorente, G., Watt, A., Salim, K., Gaskell, S. et al., Identification of early proteomic marker for hepatic steatosis. Chem. Res. Toxicol. 2006, 19, 986-998. 
[20] Strange, K., The end of "naive reductionism": Rise of systems biology or renaissance of physiology? Am. J. Physiol. Cell Physiol. 2005, 288, C968-C974.

[21] Hopkins, A. L., Groom, C. R., The druggable genome. Nat. Rev. Drug Discov. 2002, 1, 727-730.

[22] Aggarwal, K., Lee, K. H., Functional genomics and proteomics as a foundation for systems biology. Brief Funct. Genomic. Proteomic. 2003, 2, 175-184.

[23] de Hoog, C. L., Mann, M., Proteomics. Annu. Rev. Genomics Hum. Genet. 2004, 5, 267-293.

[24] Aebersold, R., Mann, M., Mass spectrometry-based proteomics. Nature 2003, 422, 198-207.

[25] Gorg, A., Weiss, W., Dunn, M. J., Current two-dimensional electrophoresis technology for proteomics. Proteomics 2004, 4, 3665-3685.

[26] Banks, R. E., Dunn, M. J., Hochstrasser, D. F., Sanchez, J. C. et al., Proteomics: New perspectives, new biomedical opportunities. Lancet 2000, 356, 1749-1756.

[27] Griffin, J. L., Understanding mouse models of disease through metabolomics. Curr. Opin. Chem. Biol. 2006, 10, 309-315.

[28] Lazar, J., Moreno, C., Jacob, H. J., Kwitek, A. E., Impact of genomics on research in the rat. Genome Res. 2005, 15, 1717-1728.

[29] Elrick, M. M., Walgren, J. L., Mitchell, M. D., Thompson, D. C., Proteomics: Recent applications and new technologies. Basic Clin. Pharmacol. Toxicol. 2006, 98, 432-441.

[30] Schuppli, C. A., Fraser, D., McDonald, M., Expanding the three Rs to meet new challenges in humane animal experimentation. Altern. Lab. Anim. 2004, 32, 525-532.

[31] Twigger, S. N., Nie, J., Ruotti, V., Yu, J. et al., Integrative genomics: In silico coupling of rat physiology and complex traits with mouse and human data. Genome Res. 2004, 14 651-660.

[32] Bogue, C. W., Genetic models in applied physiology. Functional genomics in the mouse: Powerful techniques for unraveling the basis of human development and disease. $J$. Appl. Physiol. 2003, 94, 2502-2509.

[33] Findikli, N., Candan, N. Z., Kahraman, S., Human embryonic stem cell culture: Current limitations and novel strategies. Reprod. Biomed. Online 2006, 13, 581-590.

[34] Contag, P. R., Whole-animal cellular and molecular imaging to accelerate drug development. Drug Discov. Today 2002, 7, 555-562.

[35] Caldwell, J., Problems and opportunities in toxicity testing arising from species differences in xenobiotic metabolism. Toxicol. Lett. 1992, 64, 651-659.

[36] Boyd, M. R., Grygiel, J. J., Minchin, R. F., Metabolic activation as a basis for organ-selective toxicity. Clin. Exp. Pharmacol. Physiol. 1983, 10, 87-99.

[37] Boelsterli, U. A., Ho, H. K., Zhou, S., Leow, K. Y., Bioactivation and hepatotoxicity of nitroaromatic drugs. Curr. Drug Metab. 2006, 7, 715-727.

[38] Unlu, M., Morgan, M. E., Minden, J. S., Difference gel electrophoresis: A single gel method for detecting changes in protein extracts. Electrophoresis 1997, 18, 2071-2077.

[39] Tonge, R., Shaw, J., Middleton, B., Rowlinson, R., et al., Validation and development of fluorescence two-dimensional differential gel electrophoresis proteomics technology. Proteomics 2001, 1, 377-396.
[40] Marouga, R., David, S., Hawkins, E., The development of the DIGE system: 2D fluorescence difference gel analysis technology. Anal. Bioanal. Chem. 2005, 382, 669-678.

[41] Doran, P., Martin, G., Dowling, P., Jockusch, H., Ohlendieck, K., Proteome analysis of the dystrophin-deficient MDX diaphragm reveals a drastic increase in the heat shock protein cvHSP. Proteomics 2006, 6, 4610-4621.

[42] Wittmann-Liebold, B., Graack, H. R., Pohl, T., Two-dimensional gel electrophoresis as tool for proteomics studies in combination with protein identification by mass spectrometry. Proteomics 2006, 6, 4688-4703.

[43] Wu, C. C., Yates, J. R., The application of mass spectrometry to membrane proteomics. Nat. Biotechnol. 2003, 21, 262267.

[44] Mann, M., Jensen, O. N., Proteomic analysis of post-translational modifications. Nat. Biotechnol. 2003, 21, 255-261.

[45] Celis, J. E., Honore, B., Bauw, G., Vandekerckhove, J., Comprehensive computerized 2D gel protein databases offer a global approach to the study of the mammalian cell. Bioessays $1990,12,93-97$

[46] Sanchez, J. C., Chiappe, D., Converset, V., Hoogland, C. et al., The mouse SWISS-2D PAGE database: A tool for proteomics study of diabetes and obesity. Proteomics 2001, 1, 136-163.

[47] Mulvey, C., Ohlendieck, K., Use of continuous-elution ge electrophoresis as a preparative tool for blot overlay analysis. Anal. Biochem. 2003, 319, 122-130.

[48] Kaplan, J. R., Wagner, J. D., Type 2 diabetes-an introduction to the development and use of animal models. ILAR J. 2006, 47, 181-185.

[49] Cefalu, W. T., Animal models of type 2 diabetes: Clinica presentation and pathophysiological relevance to the human condition. ILAR J. 2006, 47, 186-198.

[50] Wagner, J. E., Kavanagh, K., Ward, G. M., Auerbach, B. J. et al., Old world nonhuman primate models of type 2 diabetes mellitus. ILAR J. 2006, 47, 259-271.

[51] Bellinger, D. A., Merricks, E. P., Nichols, T. C., Swine models of type 2 diabetes mellitus: Insulin resistance, glucose tolerance, and cardiovascular complications. ILAR J. 2006, 47, 243-258.

[52] Henson, M. S., O'Brien, T. D., Feline models of type 2 diabetes mellitus. ILAR J. 2006, 47, 234-242.

[53] Engel, A. G., Franzini-Armstrong, C., Myology: Basic and Applied, 2nd edition, McGraw-Hill, New York 1994

[54] Pette, D., Historical Perspectives: Plasticity of mammalian skeletal muscle. J. Appl. Physiol. 2001, 90, 1119-1124.

[55] Carlson, B. M., Billington, L., Faulkner, J. A., Studies on the regenerative recovery of long-term denervated muscle in rats. Restor. Neurol. Neurosci. 1996, 10, 77-84.

[56] Fluck, M., Hoppeler, H., Molecular basis of skeletal muscle plasticity-from gene to form and function. Rev. Physiol. Biochem. Pharmacol. 2003, 146, 159-216.

[57] Pette, D., Staron, R. S., Transitions of muscle fiber phenotypic profiles. Histochem. Cell Biol. 2001, 115, 359-372.

[58] Ge, Y., Molloy, M. P., Chamberlain, J. S., Andrews, P. C., Proteomic analysis of mdx skeletal muscle: Great reduction of adenylate kinase 1 expression and enzymatic activity. Proteomics 2003, 3, 1895-1903. 
[59] Ge, Y., Molloy, M. P., Chamberlain, J. S., Andrews, P. C., Differential expression of the skeletal muscle proteome in $\mathrm{mdx}$ mice at different ages. Electrophoresis 2004, 25, 2576-2585.

[60] Doran, P., Dowling, P., Lohan, J., McDonnell, K. et al., Subproteomics analysis of $\mathrm{Ca}^{2+}$-binding proteins demonstrates decreased calsequestrin expression in dystrophic mouse skeletal muscle. Eur. J. Biochem. 2004, 271, 3943-3952.

[61] Doran, P., Dowling, P., Donoghue, P., Buffini, M., Ohlendieck, $\mathrm{K}$., Reduced expression of regucalcin in young and aged $\mathrm{mdx}$ diaphragm indicates abnormal cytosolic calcium handling in dystrophin-deficient muscle. Biochim. Biophys. Acta 2006, 1764, 773-785.

[62] Kanski, J., Alterman, M. A., Schoneich, C., Proteomic identification of age-dependent protein nitration in rat skeletal muscle. Free Radic. Biol. Med. 2003, 35, 1229-1239.

[63] Kanski, J., Hong, S. J., Schoneich, C., Proteomic analysis of protein nitration in aging skeletal muscle and identification of nitrotyrosine-containing sequences in vivo by nanoelectrospray ionization tandem mass spectrometry. J. Biol. Chem. 2005, 280, 24261-24266.

[64] Chang, J., Van Remmen, H., Cornell, J., Richardson, A., Ward, W. F., Comparative proteomics: Characterization of a two-dimensional gel electrophoresis system to study the effect of aging on mitochondrial proteins. Mech. Ageing Dev., 2003, 124, 33-41.

[65] Piec, I., Listrat, A., Alliot, J., Chambon, C. et al., Differential proteome analysis of aging in rat skeletal muscle. FASEB $\mathrm{J}$. $2005,19,1143-1145$

[66] Kim, N. K., Joh, J. H., Park, H. R., Kim, O. H. et al., Differential expression profiling of the proteomes and their mRNAs in porcine white and red skeletal muscles. Proteomics 2004, 4, 3422-3428

[67] Le Bihan, M. C., Hou, Y., Harris, N., Tarelli, E., Coulton, G. R. Proteomic analysis of fast and slow muscles from normal and kyphoscoliotic mice using protein arrays, 2-DE and MS. Proteomics 2006, 6, 4646-4661.

[68] Okumura, N., Hashida-Okumura, A., Kita, K., Matsubae, M. et al., Proteomic analysis of slow- and fast-twitch skeletal muscles. Proteomics 2006, 5, 2896-2906.

[69] Gelfi, C., Vigano, A., De Palma, S., Ripamonti, M. et al., 2-D protein maps of rat gastrocnemius and soleus muscles: A tool for muscle plasticity assessment. Proteomics 2006, 6, 321-340.

[70] Donoghue, P., Doran, P., Dowling, P., Ohlendieck, K., Differential expression of the fast skeletal muscle proteome following chronic low-frequency stimulation. Biochim. Biophys. Acta 2005, 1752, 166-176.

[71] Yan, J. X., Harry, R. A., Wait, R., Welson, S. Y. et al., Separation and identification of rat skeletal muscle proteins using two-dimensional gel electrophoresis and mass spectrometry. Proteomics 2001, 1, 424-434.

[72] Gelfi, C., De Palma, S., Cerretelli, P., Begum, S., Wait, R. Two-dimensional protein map of human vastus lateralis muscle. Electrophoresis 2003, 24, 286-295.

[73] Capitanio, D., Vigano, A., Ricci, E., Cerretelli, P. et al., Comparison of protein expression in human deltoideus and vastus lateralis muscles using two-dimensional gel electrophoresis. Proteomics 2005, 5, 2577-2586.

[74] Goldspink, G., Gene expression in muscle in response to exercise. J. Muscle Res. Cell Motil. 2003, 24, 121-126.
[75] Ohira, Y., Yoshinaga, T., Nomura, T., Kawano, F. et al., Gravitational unloading effects on muscle fiber size, phenotype and myonuclear number. Adv. Space Res. 2002, 30, 777-781.

[76] Chachques, J. C., Shafy, A., Duarte, F., Cattadori, B. et al., From dynamic to cellular cardiomyoplasty. J. Card. Surg. 2002, 17, 194-200.

[77] Doherty, M. K., McLean, L., Hayter, J. R., Pratt, J. M. et al., The proteome of chicken skeletal muscle: Changes in soluble protein expression during growth in a layer strain. Proteomics 2004, 4, 2082-2093.

[78] Gajendran, N., Frey, J. R., Lefkovits, I., Kuhn, L. et al., Proteomic analysis of secreted muscle components: Search for factors involved in neuromuscular synapse formation. Proteomics 2002, 2, 1601-1615.

[79] Takahashi, M., Kubota, S., Exercise-related novel gene is involved in myoblast differentiation. Biomed. Res. 2005, 26, 79-85.

[80] Forner, F., Foster, L. J., Campanaro, S., Valle, G., Mann, M., Quantitative proteomic comparison of rat mitochondria from muscle, heart, and liver. Mol. Cell. Proteomics 2006, 5, 608-619.

[81] Emery, A. E., The muscular dystrophies. Lancet 2002, 359, 687-695.

[82] Winder, S. J., Molecular Mechanisms of Muscular Dystrophies, Landes Bioscience, Georgetown, TX 2005.

[83] Watchko, J. F., O’Day, T. L., Hoffman, E. P., Functional characteristics of dystrophic skeletal muscle: Insights from animal models. J. Appl. Physiol. 2002, 93, 407-417.

[84] Durbeej, M., Campbell, K. P., Muscular dystrophies involving the dystrophin-glycoprotein complex: An overview of current mouse models. Curr. Opin. Genet. Dev. 2002, 12, 349361.

[85] Collins, C. A., Morgan, J. E., Duchenne's muscular dystrophy: Animal models used to investigate pathogenesis and develop therapeutic strategies. Int. J. Exp. Pathol. 2003, 84, 165-172.

[86] Kunkel, L. M., Bachrach, E., Bennett, R. R., Guyon, J., Steffen, L., Diagnosis and cell-based therapy for Duchenne muscular dystrophy in humans, mice, and zebrafish. J. Hum. Genet. 2006, 51, 397-406.

[87] Bulfield, G., Silver, W. G., Wight, P. A. L., Moore, K. J., Xchromosome-linked muscular dystrophy ( $\mathrm{mdx}$ ) in the mouse. Proc. Natl. Acad. Sci. USA 1984, 81, 1189-1192.

[88] Torres, L. F. B., Duchen, L. W., The mutant mdx: Inherited myopathy in the mouse. Brain 1987, 110, 269-299.

[89] Stedman, H. H., Sweeney, H. L., Shrager, J. B., Maguire, H. C. et al., The $\mathrm{mdx}$ mouse diaphragm reproduces the degenerative changes of Duchenne muscular dystrophy. Nature 1991, 352, 536-539.

[90] Sicinski, P., Geng, Y., Ryder-Cook, A. S., Barnard, E. A. et al., The molecular basis of muscular dystrophy in the $\mathrm{mdx}$ mouse: A point mutation. Science 1989, 244, 1578-1580.

[91] Ohlendieck, K., Campbell, K. P., Dystrophin-associated proteins are greatly reduced in skeletal muscle from $\mathrm{mdx}$ mice. J. Cell Biol. 1991, 115, 1685-1694.

[92] Menke, A., Jockusch., H., Decreased osmotic stability of dystrophin-less muscle cells from mdx mouse. Nature 1991, $349,69-71$. 
[93] Lynch, G. S., Rafael, J. A., Chamberlain, J. S., Faulkner, J. A., Contraction-induced injury to single permeabilized muscle fibers from $\mathrm{mdx}$, transgenic $\mathrm{mdx}$, and control mice. Am. J. Physiol. Cell Physiol. 2000, 279, C1290-C1294.

[94] DeLuca, A., Pierno, S., Liantonio, A., Cetrone, M. et al., Alteration of excitation-contraction coupling mechanism in extensor digitorum longus muscle fibres of dystrophic $\mathrm{mdx}$ mouse and potential efficacy of taurine. Br. J. Pharmacol. 2001, 132, 1047-1054.

[95] Culligan, K., Banville, N., Dowling, P., Ohlendieck, K., Drastic reduction of calsequestrin-like proteins and impaired calcium binding in dystrophic mdx muscle. J. Appl. Phy siol. 2002, 92, 435-445.

[96] Dowling, P., Doran, P., Ohlendieck, K., Drastic reduction of sarcalumenin in Dp427 (dystrophin of $427 \mathrm{kDa}$ )-deficient fibres indicates that abnormal calcium handling plays a key role in muscular dystrophy. Biochem. J. 2004, 379, 479-488.

[97] Dowling, P., Lohan, J., Ohlendieck, K., Comparative analysis of Dp427-deficient mdx tissues shows that the milder dystrophic phenotype of extraocular and toe muscle fibres is associated with a persistent expression of beta-dystroglycan. Eur. J. Cell Biol. 2003, 82, 222-230.

[98] Dowling, P., Culligan, K., Ohlendieck, K., Distal mdx muscle groups exhibiting up-regulation of utrophin and rescue of dystrophin-associated glycoproteins exemplify a protected phenotype in muscular dystrophy. Naturwissenschaften, 2002, 89, 75-78.

[99] Dowling, P., Doran, P., Lohan, J., Culligan, K., Ohlendieck, K., Naturally protected muscle phenotypes: Development of novel treatment strategies for Duchenne muscular dystrophy. Basic Appl. Myol. 2004, 14, 169-178.

[100] Ohlendieck, K., Ervasti, J. M., Matsumura, K., Kahl, S. D. et al., Dystrophin-related protein is localized to neuromuscular junctions of adult skeletal muscle. Neuron 1991, 7, 499-508.

[101] Porter, J. D., Rafael, J. A., Ragusa, R. J., Brueckner, J. K. et al., The sparing of extraocular muscle in dystrophinopathy is lost in mice lacking utrophin and dystrophin. J. Cell Sci. 1998, 111, 1801-1811.

[102] Deconinck, N., Tinsley, J., De Backer, F., Fisher, R. et al., Expression of truncated utrophin leads to major functional improvements in dystrophin-deficient muscles of mice. Nat. Med. 1997, 3, 1216-1221.

[103] Tinsley, J., Deconinck, N., Fisher, R., Kahn, D. et al. Expression of full-length utrophin prevents muscular dystrophy in mdx mice. Nat. Med. 1998, 4, 1441-1444.

[104] Wakefield, P. M., Tinsley, J. M., Wood, M. J., Gilbert, R. et al., Prevention of the dystrophic phenotype in dystrophin/ utrophin-deficient muscle following adenovirus-mediated transfer of a utrophin minigene. Gene Ther. 2000, 7, 201204.

[105] Miura, P., Jasmin, B. J., Utrophin upregulation for treating Duchenne or Becker muscular dystrophy: How close are we? Trends Mol. Med. 2006, 12, 122-129.

[106] Ryan, M., Butler-Browne, G., Erzen, I., Mouly, V. et al., Persistent expression of the alpha1S-dihydropyridine receptor in aged human skeletal muscle: Implications for the excitation-contraction uncoupling hypothesis of sarcopenia. Int. J. Mol. Med. 2003, 11, 425-434.
[107] Ryan, M., Carlson, B. M., Ohlendieck, K., Oligomeric status of the dihydropyridine receptor in aged skeletal muscle. Mol. Cell. Biol. Res. Commun. 2001, 4, 224-229.

[108] Lingelbach, L. B., McDonald, R. B., Description of the longterm lipogenic effects of dietary carbohydrates in male Fischer 344 rats. J. Nutr. 2000, 130, 3077-3084

[109] Fernandes, G., Venkatraman, J. T., Turturro, A., Attwood, V. G., Hart, R. W., Effect of food restriction on life span and immune functions in long-lived Fischer-344 x Brown Norway F1 rats. J. Clin. Immunol. 1997, 17, 85-95.

[110] Isfort, R. J., Wang, F., Greis, K. D., Sun, Y. et al., Proteomic analysis of rat soleus muscle undergoing hindlimb suspension-induced atrophy and reweighting hypertrophy. Proteomics 2002, 2, 543-550.

[111] Isfort, R. J., Wang, F., Greis, K. D., Sun, Y. et al., Proteomic analysis of rat soleus and tibialis anterior muscle following immobilization. J. Chromatogr. B 2002, 769, 323-332.

[112] Isfort, R. J., Proteomic analysis of striated muscle. J. Chromatogr. 2002, B771, 155-165.

[113] Koenig, M., Hoffman, E. P., Bertelson, C. J., Monaco, A. P. et al., Complete cloning of the Duchenne muscular dystrophy (DMD) cDNA and preliminary genomic organization of the DMD gene in normal and affected individuals. Cell 1987, 50, 509-517.

[114] Emery, A., Muntoni, F., Duchenne Muscular Dystrophy, 3rd edition, Oxford University Press, Oxford 2003.

[115] Tkatchenko, A. V., Le Cam, G., Leger, J. J., Dechesne, C. A., Large-scale analysis of differential gene expression in the hindlimb muscles and diaphragm of $\mathrm{mdx}$ mouse. Biochim. Biophys. Acta. 2000, 1500, 17-30.

[116] Chen, Y. W., Zhao, P., Borup, R., Hoffman, E. P., Expression profiling in the muscular dystrophies: identification of novel aspects of molecular pathophysiology. J. Cell Biol. 2000, 151, 1321-1336.

[117] Porter, J. D., Khanna, S., Kaminski, H. J., Rao, J. S. et al., A chronic inflammatory response dominates the skeletal muscle molecular signature in dystrophin-deficient $m d x$ mice. Hum. Mol. Genet. 2002, 11, 263-272.

[118] Haslett, J. N., Sanoudou, D., Kho, A. T., Bennett, R. R. et al., Gene expression comparison of biopsies from Duchenne muscular dystrophy (DMD) and normal skeletal muscle. Proc. Natl. Acad. Sci. USA 2003, 99, 15000-15005.

[119] Porter, J. D., Merriam, A. P., Leahy, P., Gong, B., Khanna, S., Dissection of temporal gene expression signatures of affected and spared muscle groups in dystrophin-deficient ( $m d x)$ mice. Hum. Mol. Genet. 2003, 12, 1813-1821.

[120] Turk, R., Sterrenburg, E., de Meijer, E. J., van Ommen, G-J. B. et al., Muscle regeneration in dystrophin-deficient $m d x$ mice studied by gene expression profiling. BMC Genomics $2005,6,98$.

[121] Baker, P. E., Kearney, J. A., Gong, B., Merriam, A. P. et al., Analysis of gene expression differences between utrophin/ dystrophin-deficient vs $m d x$ skeletal muscles reveals a specific upregulation of slow muscle genes in limb muscles. Neurogenetics 2006, 7, 81-91.

[122] Ohlendieck, K., Towards an understanding of the dystrophin-glycoprotein complex: Linkage between the extracellular matrix and the subsarcolemmal membrane cytoskeleton. Eur. J. Cell Biol. 1996, 69, 1-10. 
[123] Culligan, K. G., Mackey, A. J., Finn, D. M., Maguire, P. B. et al., Role of dystrophin isoforms and associated proteins in muscular dystrophy (review). Int. J. Mol. Med. 1998, 2, 639648

[124] Batchelor, C. L., Winder, S. J., Sparks, signals and shock absorbers: How dystrophin loss causes muscular dystrophy. Trends Cell Biol. 2006, 16, 198-205.

[125] Krief, S., Faivre, J. F., Robert, P., le Douarin, B. et al., Identification and characterization of cvHSP. A novel human small stress protein selectively expressed in cardiovascular and insulin-sensitive tissues. J. Biol. Chem. 1999, 274, 36592-36600

[126] de Jong, W. W., Leunissen, J. A., Voorter, C. E., Evolution of the alpha-crystallin/small heat shock protein family. Mol. Biol. Evol. 1993, 10, 103-126.

[127] Nicholl, I. D., Quinlan, R. A., Chaperone activity of alphacrystallins modulates intermediate filament assembly EMBO J. 1994, 13, 945-953.

[128] Bouchentouf, M., Benabdallah, B. F., Tremblay, J. P., Myoblast survival enhancement and transplantation success improvement by heat-shock treatment in $\mathrm{mdx}$ mice. Transplantation 2004, 77, 1349-1356.

[129] Borman, L., Polla, B. S., Lotz, B. P., Gericke, G. S., Expression of heat shock/stress proteins in Duchenne muscular dystrophy. Muscle Nerve 1995, 18, 23-31.

[130] Ohlendieck, K., in: Winder, S. J. (Ed.), Molecular Mechan isms of Muscular Dystrophies, Landes Bioscience, Georgetown, TX 2005, pp. 188-197.

[131] Alderton, J. M., Steinhardt, R. A., Calcium influx through calcium leak channels is responsible for the elevated levels of calcium-dependent proteolysis in dystrophic myotubes. J. Biol. Chem. 2000, 275, 9452-9460.

[132] Mallouk, N., Jacquemond, V., Allard, B., Elevated subsarcolemmal $\mathrm{Ca}^{2+}$ in $\mathrm{mdx}$ mouse skeletal muscle fibres detected with $\mathrm{Ca}^{2+}$-activated $\mathrm{K}^{+}$channels. Proc. Natl. Acad. Sci. USA 2000, 97, 4950-4955.

[133] Berchtold, M. W., Brinkmeier, H., Muntener, M., Calcium ion in skeletal muscle: Its crucial role for muscle function, plasticity, and disease. Physiol. Rev. 2000, 80, 1215-1265.

[134] Navarro, A., Lopez-Cepero, J. M., Sanchez del Pino., M, J., Skeletal muscle ageing. Front. Biosci. 2001, 6, 26-44.

[135] Carmeli, E., Coleman, R., Reznick, A. Z., The biochemistry of aging muscle. Exp. Gerontol. 2002, 37, 477-489.

[136] Luff, A. R., Age-associated changes in the innervation of muscle fibres and changes in the mechanical properties of motor units. Ann. NYAcad. Sci. 1998, 854, 92-101.
[137] Larsson, L., The age-related motor disability: Underlying mechanisms in skeletal muscle at the motor unit, cellular and molecular level. Acta Physiol. Scand. 1998, 163, S27S29.

[138] Carlson, B. M., Denervation and the aging of skeletal muscle. Bas. Appl. Myol. 2004, 14, 135-140.

[139] Dirks, A, Leeuwenburgh, C., Apoptosis in skeletal muscle with aging. Am. J. Physiol. 2002, 282, R519-R527.

[140] Balagopal, P., Rooyackers, O. E., Adey, D. B., Ades, P. A., Nair, K. S., Effects of aging on in vivo synthesis of skeletal muscle myosin heavy-chain and sarcoplasmic protein in humans. Am. J. Physiol. 1997, 273, E790-E800.

[141] Ryan, M., Ohlendieck, K., Excitation-contraction uncoupling and sarcopenia. Bas. Appl. Myol. 2004, 14, 141-154.

[142] Roberts, S. B., Effects of aging on energy requirements and the control of food intake in men. J. Gerontol. 1995, 50A, 101-106.

[143] Degens, H., Age-related changes in the microcirculation of skeletal muscle. Adv. Exp. Med. Biol. 1998, 454, 343-348.

[144] Bua, E. A., McKiernan, S. H., Wanagat, J., McKenzie, D., Aiken, J. M., Mitochondrial abnormalities are more frequent in muscles undergoing sarcopenia. J. Appl. Physiol. 2002, 92, 2617-2624.

[145] Renault, V., Thornell, L. E., Eriksson, P. O., Butler-Browne, G., Mouly, V., Regenerative potential of human skeletal muscle during aging. Aging Cell 2002, 1, 132-139.

[146] Ahn, A. H., Kunkel, L. M., The structural and functional diversity of dystrophin. Nat. Genet. 1993, 3, 283-291.

[147] Deconinck, N., Dan, B., Pathophysiology of Duchenne muscular dystrophy: Current hypotheses. Pediatr. Neurol. 2007, 36, 1-7.

[148] Arab, S., Gramolini, A. O., Ping, P., Kislinger, T. et al., Cardiovascular proteomics: Tools to develop novel biomarkers and potential applications. J. Am. Coll. Cardiol. 2006, 48, 1733-1741.

[149] van der Merwe, D. E., Oikonomopoulou, K., Marshall, J. Diamandis, E. P., Mass spectrometry: Uncovering the cancer proteome for diagnostics. Adv. Cancer Res. 2007, 9, 2350 .

[150] Fountoulakis, M., Kossida, S., Proteomics-driven progress in neurodegeneration research. Electrophoresis 2006, 27, 1556-1573. 